\title{
The value of radiology in predicting gallstone type when selecting patients for medical treatment
}

\author{
G. D. BELL ${ }^{1}$, R. H. DOWLING 2 , B. WHITNEY ${ }^{3}$, AND D. J. SUTOR \\ From the Departments of Medicine and Radiodiagnosis, Royal Postgraduate Medical School, London, and \\ the Department of Chemistry, University College, London
}

SUMMARY Since medical treatment of gallstones is confined to cholesterol-rich stones, the ability of clinical radiographs to predict gallstone type was tested prospectively by comparing the preoperative radiological appearance of gallstones from 57 unselected patients with cholelithiasis coming to cholecystectomy with the subsequent analysis of the stones both by $x$-ray diffraction and by chemical techniques. Fifty-two per cent of the patients had 'non-functioning' gallbladders which failed to opacify after at least two contrast examinations and 25 out of 50 had radioopaque stones. Of the 25 patients with radiolucent stones, the stones in $20(80 \%)$ were predominantly cholesterol in type but radiology was misleading in five; three contained $40-55 \%$ calcium salts but were still radiolucent while two were amorphous and contained $<10 \%$ cholesterol by weight on chemical analysis. While radiology was sometimes misleading when the stones were small and irregular, large radiolucent stones with a smooth profile were invariably chloesterol-rich stones. The results also show that in men calcified stones were commoner than in women and that in older women the gallstones contained more calcium salts and less cholesterol than in younger women $<50 \mathrm{yr}$ ). This paper analyses critically the value and limitations of clinical radiology in predicting gallstone type.

In the past two years, studies from the Mayo Clinic (Thistle and Schoenfield, 1971; Danzinger, Hofmann, Schoenfield, and Thistle, 1972; Thistle and Hofmann, 1973), our own unit (Bell, Whitney, and Dowling, 1972a; Mok, Bell, and Dowling, 1974), and elsewhere (James, Cullen, and Bouchier, 1973; Hofmann and Paumgartner, 1974; Beker, 1974; Coyne, Bonorris, Goldstein, Lahana, and Schoenfield, 1974; Moeckel, 1974) have shown that treatment with oral chenodeoxycholic acid (CDCA) improves cholesterol solubility in bile and, as a result, dissolves cholesterol gallstones. However, preliminary results suggest that CDCA is ineffective for calcified radioopaque stones (Bell et al, 1972a; Thistle and Hofmann, 1973) and to date there is no iknown way of 'dissolving' non-cholesterol gallstones containing calcium salts and bile pigments (Sutor and Wooley, 1973, 1974a and b). The selection of

\footnotetext{
${ }^{1}$ Present address: Department of Medicine, St Bartholomew's Hospital, London, EC1.

${ }^{2}$ Request for reprints to R.H.D. at Gastroenterology Unit, Department of Medicine, Guy's Hospital Medical School, London, SE1 9RT.

'Present address: St Mary's General Hospital, Milton, Portsmouth, PO3 6AD.

Received for publication 3 March 1975.
}

patients with gallstones suitable for dissolution therapy is therefore of considerable importance. At present, this selection is based on the radiological appearance of the stones, both on plain radiographs and at cholecystography, but surprisingly little is known about the accuracy with which the composition of any given gallstone may be predicted from clinical radiographs.

In this prospective study, we have correlated the preoperative radiological appearance of gallstones from 57 patients undergoing elective cholecystectomy with the composition of the stones, removed at operation, as measured both by $x$-ray diffraction and by chemical analysis. We have also examined the influence of age and sex on the type of gallstone present. This paper reports our findings.

\section{Materials and Methods}

THE GALLSTONES

Gallstones were obtained from 57 patients (42 women and 15 men; mean age 50.9; age range 25-86) undergoing elective cholecystectomy. Apart from the clinical indications which led to surgery, patients were not selected. 
The stones from all 57 patients were analysed by $x$-ray diffraction. In 50 of these patients clinical radiographs were available for comparison. In addition, the stones from 50 of the 57 patients were analysed chemically, and, of these, clinical radiographs were available in 47.

\section{X-ray diffraction}

The gallstones were soaked in $10 \%$ aqueous formalin for 24 hours, washed well with water, and air dried. They were then cut in half and, where possible, samples taken from the nucleus, interior, and surface of the stone for analysis by the $x$-ray powder method (Sutor and Wooley, 1969; 1971). This method has shown that the following crystalline compounds can be identified in gallstones-cholesterol monohydrate and two forms of anhydrous cholesterol, three forms of calcium carbonate (aragonite, vaterite, and calcite), two of calcium phosphate (apatite and whitlockite), and calcium palmitate. The technique cannot usually be used to identify amorphous material. Its accuracy varies considerably depending on the type of stone analysed but in our hands, the maximum variation was estimated to be $\pm 12 \%$.

\section{Chemical analysis}

The split gallstones were finely ground with a pestle and mortar, and the resultant powder dried at $50^{\circ} \mathrm{C}$ for seven days. After thorough mixing, $20 \mathrm{mg}$ aliquants of the dried powder were extracted with $3 \times$ $15 \mathrm{ml}$ washes of acidified chloroform:methanol $(2: 1 \mathrm{v} / \mathrm{v})$ and the volume of the pooled extracts made up to $50 \mathrm{ml}$ with the extraction mixture. Pilot studies were then carried out to see whether or not it was necessary to split and separate the phases in the chloroform:methanol mixture before the cholesterol estimations. In the first eight samples, either $2.5 \mathrm{ml}$ of the 'unsplit' chloroform:methanol mixture was evaporated to dryness in a rotary evaporator and the residue redissolved in $0.5 \mathrm{ml}$ glacial acetic acid, or the phases in the mixture were first split by adding $20 \%$ by volume water, before taking the $2.5 \mathrm{ml}$ aliquant from the chloroform phase for processing as above. The cholesterol content, estimated with the modification by Carr and Drekter (1956) of the method of Abell, Levy, Brodie, and Kendall (1952) in the glacial acetic acid extracts, was essentially the same in both methods $(74.3 \pm 10 \cdot 5 \%$ in the unsplit extracts compared with $74.1 \pm 10.6 \%$ in the chloroform phase only). For this reason, the cholesterol content of all the remaining stones was estimated in the unsplit, chloroform:methanol extracts and the percentage cholesterol by weight in the dried powdered stones calculated. This method was modified from that described by Nakayama (1968).

When the reproducibility of the method was also checked with duplicate determinations on eight samples analysed on two separate occasions, the coefficient of variation was $2 \cdot 14 \%$.

\section{Clinical radiology}

Of the 50 patients whose clinical radiographs were available, three had only plain radiographs of the gallbladder area, while 47 had oral cholecystograms, and, in some cases, intravenous cholangiograms. Gallbladders which opacified and contracted well in response to a fatty meal were considered as 'functioning' while those which failed to opacify after a minimum of two contrast examinations were classified as 'non-functioning'. Before knowing the results of the stone analyses, the radiographs were examined by one of us (B.W.) and the gallstones classified as radiolucent or radioopaque. The radioopaque stones were then subclassified on an arbitrary scale from + for stones which were only very faintly opaque to ++++ for stones which were densely calcified: radiolucent stones were graded as 0 .

Although the main purpose of this paper was to examine the reliability of clinical radiographs in predicting the composition of a given gallstone, the results have also provided information about the chemical and crystallographic composition of gallstones in a relatively unselected population of patients and about the influence of age and sex on the type of gallstones found. These data are presented first before comparing the results obtained with the different methods.

\section{Results}

GALLSTONE COMPOSITION BY X-RAY DIFFRACTION

\section{Crystalline composition of the gallstones}

The results of the $x$-ray diffraction studies are shown in table I. The mean weighted percentage composition (Sutor and Wooley, 1971) of cholesterol in the gallstones from the 57 patients was 63.4 ( \pm SEM $5.0) \%$ of the total crystalline material. Calcium salts made up $31.8( \pm 4.6) \%$ and the remaining $4.8 \%$ was amorphous material. Calcium carbonate contributed $54.7 \%$ of the total calcium salts, calcium phosphate $39 \cdot 3$, and calcium palmitate only $6 \%$.

In general, gallstones from women contained more cholesterol than those from men, while in younger women (aged less than 50 years) the gallstones contained approximately $15 \%$ more cholesterol than did the stones in the older female group. This $15 \%$ difference was partly due to lesser amounts of calcium salts, but was mainly due to the fact that in the older women the stones contained $10 \%$ more amorphous material (table I). 


\begin{tabular}{|c|c|c|c|c|c|c|}
\hline Group & Number & $\begin{array}{l}\text { Crystalline } \\
\text { Cholesterol }\end{array}$ & $\begin{array}{l}\text { Calcium Carbonate } \\
\text { Compounds }\end{array}$ & $\begin{array}{l}\text { Calcium Phosphate } \\
\text { Compounds }\end{array}$ & $\begin{array}{l}\text { Calcium } \\
\text { Palmitate }\end{array}$ & $\begin{array}{l}\text { Amorphous material } \\
(\%) \text { (approximate } \\
\text { values) }\end{array}$ \\
\hline
\end{tabular}

Table I Crystalline composition of gallstones from 57 patients as determined by $\mathrm{x}$-ray diffraction (mean values $\pm S E M)$

The predominance of different gallstone types

While table I shows the relative proportions of the different crystalline substances present, table II shows the types of gallstones which were classified arbitrarily as pure cholesterol stones ( $>95 \%$ cholesterol), mixed stones (containing different forms of cholesterol, calcium salts and bile pigments) which have been subdivided into cholesterol-rich stones (> 70\% of cholesterol) and stones containing $<70 \%$ cholesterol; stones of calcium salts, and amorphous stones.

Taking the results for men and women together, $60 \%$ of the stones were predominantly cholesterol in type, $23 \%$ were mixed stones containing less than $70 \%$ cholesterol, while $14 \%$ of the stones were made of crystals of calcium salts. Considering the findings for the various subgroups separately, there were almost twice as many calcium-rich stones in men as in women, while in the older women, both amorphous stones and stones of calcium salts were commoner, although these observations are based on small sample numbers.

\section{CHEMICAL ANALYSIS}

\section{Predominance of cholesterol-rich stones}

Sixty-eight per cent of the 50 stones analysed chemically contained more than $70 \%$ cholesterol by weight.

Comparison between cholesterol content of stones measured both chemically and by $\mathrm{x}$-ray diffraction Taking the results for all 50 stones together (includ- ing those for mixed stones and for stones of calcium salts, as defined by $x$-ray diffraction), the mean cholesterol content, measured chemically, was 72.7 ( \pm SEM $5 \cdot 3$ ) $\%$ by weight, compared with $68 \cdot 3 \pm$ $5.0 \%$ when the same 50 stones were analysed by the diffraction technique. The correlation between the results of analyses of these 50 stones by the two

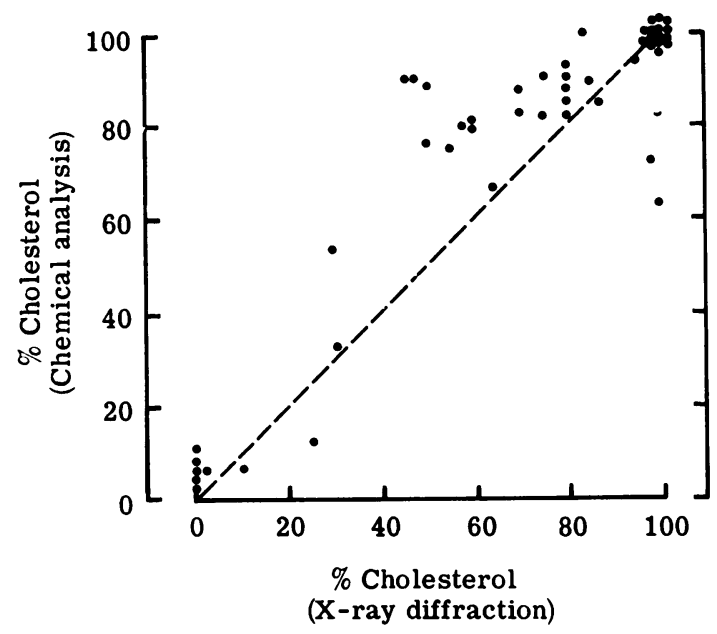

Fig 1 Relationship between the cholesterol content of 50 gallstones as estimated both chemically and by the $\mathrm{x}$-ray diffraction technique (see text). The broken $45^{\circ}$ line is the line of identity showing that the amount of cholesterol estimated chemically was generally greater than that found by the diffraction method.

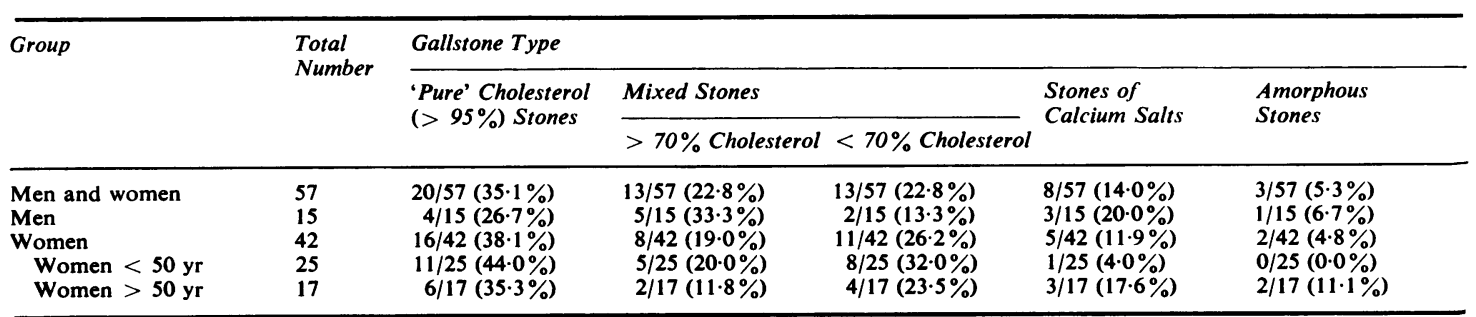

Table II Frequency of different types of gallstones as determined by $\mathrm{x}$-ray diffraction ${ }^{1}$

'The amorphous stones were recognized by the absence of a powder diffraction pattern although they do yield a non-powder 'amorphous' diffraction pattern. 
methods is shown in figure 1 . There was reasonable agreement between the two techniques, particularly for the 'pure' cholesterol stones. In the case of mixed stones, however, the amount of cholesterol estimated chemically was generally greater than that measured by $x$-ray diffraction, while stones which contained no cholesterol, as defined by the crystallographic technique, contained as much as $5-10 \%$ cholesterol on chemical analysis. Because the results of these $x$-ray diffraction studies were not accurately quantitative, it was not possible to determine a correlation coefficient between the results obtained using the two analytical methods.

PREDICTION OF GALLSTONE TYPE BY CLINICAL RADIOGRAPHS

Relationship between the radioopacity of gallstones and their $\mathrm{x}$-ray diffraction analysis

The comparison between the arbitrary grading of radioopacity (from clinical radiographs) with the $x$-ray diffraction analysis in $\mathbf{4 8}$ of the 50 gallstones ${ }^{1}$ so studied, is illustrated in figure 2 . As indicated by the double abscissa, the $x$-ray diffraction studies showed that there was a roughly reciprocal relationship between the percentage of calcium salts and the percentage of crystalline cholesterol in the individual stones. Surprisingly, half of the 50 stones were radioopaque and, in general, the greater the percentage of calcium salts, the more radioopaque were the stones. Conversely, the majority (but not all) of the radiolucent stones were rich in cholesterol with little or no

'Two of the 50 stones contained only amorphous material and therefore did not yield a powder diffraction pattern.

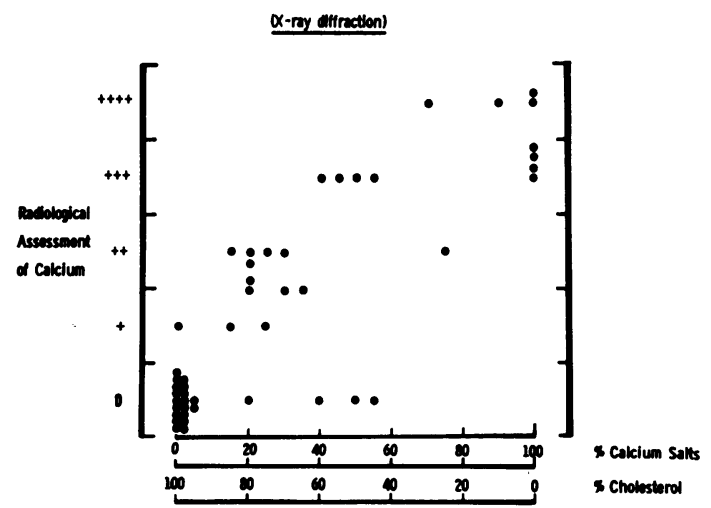

Fig 2 Relationship between the radioopacity of 50 gallstones, as assessed by clinical radiography and graded arbitrarily on the ordinate from 0 , radiolucent, to ++++ , densely radioopaque, and the percentage of calcium and cholesterol in the stones shown as reciprocals on the double abscissa. calcium salts. Of the 25 radiolucent stones, 20 contained more than $70 \%$ cholesterol, but in five, any assumption that radiolucent stones may be equated with cholesterol-rich stones would have been misleading. Three of these stones contained between $\mathbf{4 0}$ and $55 \%$ calcium salts, while the two amorphous stones contained less than $10 \%$ cholesterol on chemical analysis.

Relationship between radiolucency of gallstones and their cholesterol content as measured chemically

Of the 47 patients in whom both clinical radiographs and chemical analyses of stones were available, 24 stones were radiolucent and 23 radioopaque. The mean cholesterol content of the radiolucent stones was $86.2 \pm$ SEM $5 \cdot 2 \%$ (range 6-100) compared with $59 \cdot 0 \pm 7 \cdot 3 \%$ (range $3-98$ ) in the radioopaque group and this difference was statistically significant $(t=$ 3.07; $P<0.025$ ).

\section{GALLBLADDER FUNCTION AND GALLSTONE TY PE}

In this particular series of 47 patients, who came to surgery and who had had both oral cholecystograms and/or intravenous cholangiograms, only $22(47 \%)$ had radiologically 'functioning' gallbladders and of these 22 patients, only nine had radiolucent gallstones.

\section{Discussion}

Since the successful treatment of cholelithiasis with CDCA seems confined to cholesterol-rich gallstones, the ability to select patients with this type of stones is obviously of considerable importance. Indeed, when gallstone dissolution was studied in vivo, in bile fistula rhesus monkeys, the rate of dissolution seemed proportional to the cholesterol content of the stones (Bell, Sutor, Whitney, and Dowling, 1972b). The results of the present paper suggest that clinical radiographs provide a reasonably reliable, but not completely accurate, indication of the type of gallstone present. It seems that about one patient in five with radiolucent stones has gallstones which are not predominantly cholesterol in type. This $20 \%$ incidence of 'false positives' was mainly found in patients with small, irregular, radiolucent stones which subsequently proved to be non-cholesterolrich stones; large radiolucent stones with a smooth contour were invariably cholesterol-rich stones.

There have been few other studies relating the radiological appearance of gallstones to their subsequent analysis. Since our preliminary report on this subject (Bell, Sutor, and Dowling, 1973), Trotman, Petrella, Soloway, Sanchez, Morris, and Miller (1974) have also compared preoperative 
clinical radiographs with post-cholecystectomy analysis of gallstones from 79 patients. They showed that $19 \%$ of 63 radiolucent gallstones were noncholesterol in type-a figure which agrees well with the present study-while four of 16 radioopaque stcnes were cholesterol in type. Based on studies for up to 25 years, Wolpers (1974) has also defined the radiological criteria for distinguishing between cholesterol and pigment stones.

In general, the present study shows that there was close agreement between the results obtained by the two techniques used to measure the cholesterol content of the gallstones. However, the values for the percentage of cholesterol measured chemically were usually slightly higher than those estimated by $x$-ray diffraction, and this was particularly true for stones of mixed composition which contained appreciable amounts of calcium. There are several possible reasons for this apparent discrepancy. First, the $x$ ray diffraction technique measures only crystalline material, and therefore in stones which contain amorphous cholesterol, the crystallographic technique underestimates the amount of cholesterol present. Second, only a few milligrams of material are used in the powder method, but to minimize any possible sampling error, several such samples were selected so as to be representative of the composition of the stone as determined by microscopic examination. However, not all of the stone was crushed and examined, and hence some constituents might occasionally be missed. Third, in mixtures of calcium salts and cholesterol, the calcium salt absorbs the diffracted rays of cholesterol and vice versa, but the interference by calcium salts is very much stronger than that caused by cholesterol. For this reason, in the presence of calcium salts, small amounts of up to 5 to $7 \%$ of cholesterol may not be detected by the diffraction technique. It must also be remembered that the two analyses were performed either on different halves of the same stone or on 'sister' stones and are therefore subject to another sort of sampling error.

The $50 \%$ incidence of radioopaque gallstones found in the present study is considerably higher than the 5 to $20 \%$ incidence usually quoted in other series (Rains, 1964; Shanks and Kerley, 1969; Salzman, 1966; Shedadi, 1966) although Sutton (1969) described a $40 \%$ incidence of opaque stones. While an attempt was made to avoid selection in the present study, nonetheless the patients had all come to cholecystectomy, mainly as an elective operation. It could be argued that the high incidence of radioopaque stones was due to the fact that opaque stones are more likely to be detected and therefore the patient be referred to surgery.
The gallstones in this study were only classified into two groups, radiolucent and radioopaque. However, radioopaque stones do not form a homogeneous group and their opacity depends on the distribution and density of calcium salts within the stone. The present series included stones which were heavily, and those which were faintly, calcified, stones in which the calcium was uniformly distributed, and those in which there was a patchy calcification and stones in which there was either a calcified nucleus, concentric laminations of calcification, or a calcified rim. The frequency of radioopaque stones in a completely unselected population may therefore be less than that found here. On the other hand, the presence of soft tissue on abdominal radiographs may mask the presence of small amounts of calcium and stones which appear radiolucent in vivo are often radioopaque when radiographed outside the body. In general, however, medium sized (arbitrarily defined as measuring 5 to $10 \mathrm{~mm}$ across) and large ( $>10 \mathrm{~mm})$ stones containing more than 10 to $20 \%$ calcium salts, as measured by $x$-ray diffraction, were opaque on clinical radiographs. The three radiolucent stones which contained $40-55 \%$ calcium salts, as measured by the diffraction technique, all small stones ( $<5 \mathrm{~mm}$ in diameter).

The relatively high incidence of patients with nonfunctioning gallbladders may also be due to the fact that the patients had all come to surgery. Gough and Bennett (1971) suggested that there was a relationship between the cholecystographic findings and the duration of symptoms in cholelithiasis and that with time there was a progressive change in the cholecystogram from functioning to non-functioning gallbladders. However, Moeckel (1974) has described two patients who had non-opacifying gallbladders before treatment which became normal during therapy with chenodeoxycholic acid.

The present results have also provided information about the influence of age and sex on the type of gallstones found in patients coming to cholecystectomy. In general, these results confirm the previous findings of Sutor and Wooley (1971) that gallstones formed by men tend to contain more calcium salts and less cholesterol than those formed by women. In female patients, the incidence of both pure calcium stones and amorphous stones was higher in the older age group. Furthermore, the mean percentage of calcium phosphate, as determined by the $x$-ray diffraction technique in the present series, was much higher than that previously reported by Sutor and Wooley (1971) but the reason for this difference is not clear.

Although epidemiological studies can provide statistical information about the likelihood of cholesterol stones in different populations and the 
demonstration that fasting gallbladder bile-rich duodenal fluid is supersaturated with cholesterol, and contains cholesterol microcrystals, may provide supportive but non-specific evidence of cholesterol stones, at present clinical radiographs provide the most reliable index of gallstone type in a given patient.

\section{References}

Abell, L. L., Levy, B. B., Brodie, B. B., and Kendall, F. E. (1952). A simplified method for the estimation of total cholesterol in serum and demonstration of its specificity. J. biol. Chem., 195, 357-366.

Beker, S. (1974). Ensayo terapeutico con el acido quenodeoxicolico para disolver calculos biliares ('colesterol'). (Abstr.) In Proceedings of the Sth World Congress of Gastroenterology, Mexico City, 1974, p. 481.

Bell, G. D., Sutor, D. J., and Dowling, R. H. (1973). Relationship between bile and gallstone composition to the rate of gallstone dissolution in the rhesus monkey and in man. Biol. Gastroent. (Paris), 6, 172-173.

Bell, G. D., Sutor, D. J., Whitney, B., and Dowling, R. H. (1972b). Factors influencing human gallstone dissolution in monkey, dog and human bile (Abstr.). Gut, 13, 836.

Bell, G. D., Whitney, B., and Dowling, R. H. (1972a). Gallstone dissolution in man using chenodeoxycholic acid. Lancet, 2, 1213-1216.

Carr, J. J., and Drekter, I. J. (1956). Simplified rapid technic for the extraction and determination of serum cholesterol without saponification. Clin. Chem., 2, 353-368.

Coyne, M. J., Bonorris, G. G., Goldstein, L. I., Lahana, D. A., and Schoenfield, L. J. (1974). Dissolution of gallstones by chenodeoxycholic acid (CDC) and phenobarbital (PB). (Abstr.). Gastroenterology, 66, 679.

Danzinger, R. G., Hofmann, A. F., Schoenfield, L. J., and Thistle, J. L. (1972). Dissolution of cholesterol gallstones by chenodeoxycholic acid. New Engl. J. Med., 286, 1-8.

Gough, W. W., and Bennett, P. H. (1971). Cholecystographic findings: Relation to duration and symptomatology of cholelithiasis. (Abstr.). Gastroenterology, 60, 743.

Hofmann, A. F., and Paumgartner, G., Eds. (1974). Chenodeoxycholic Acid Therapy of Gallstones. Proceedings of a Workshop held in Freiburg i. Br., 1973. Schattauer, Stuttgart.
James, O., Cullen, J., and Bouchier, I. A. D. (1973). A study of the effectiveness, toxicity, and bile salt kinetics of chenodeoxycholic acid in the treatment of human gallstones. (Abstr.) Gut, 14, 827.

Moeckel, G. (1974). Gallstone dissolution in man with and without chenodeoxycholic acid therapy. (Abstr.). In Proceedings of the Sth World Congress of Gastroenterology, Mexico City, 1974, p. 516.

Mok, H. Y. I., Bell, G. D., and Dowling, R. H. (1974). Effect of different doses of chenodeoxycholic acid on bile-lipid composition and on frequency of side-effects in patients with gallstones. Lancet, 2, 253-257.

Nakayama, F. (1968). Quantitative microanalysis of gallstones. J. Lab. clin. Med., 72, 602-611.

Rains, A. J. H. (1964). Gallstones: Causes and Treatment, p. 121. Heinemann, London.

Salzman, E. (1966). Opacification of bile duct calculi. Radiol. Clin. N. Amer., 4, 525-533.

Shanks, S. C., and Kerley, P., Eds. (1969). A Textbook of X-Ray Diagnosis, vol. 4, p. 546. Lewis, London.

Shehadi, W. H. (1966). Radiologic examination of the biliary tract: plain film of the abdomen; oral cholecystography. Radiol. Clin. N. Amer., 4, 463-482 (see p. 471).

Sutor, D. J., and Wooley, S. E. (1969). X-ray diffraction studies of the composition of gallstones from English and Australian patients. Gut, 10, 681-683.

Sutor, D. J., and Wooley, S. E. (1971). A statistical survey of the composition of gallstones in 8 countries. Gut, 12, 55-64.

Sutor, D. J., and Wooley, S. E. (1973). The nature and incidence of gallstones containing calcium. Gut, 14, 215-220.

Sutor, D. J., and Wooley, S. E. (1974a). The sequential deposition of crystalline material in gallstones: Evidence for changing gallbladder bile composition during the growth of some stones. Gut, 15, 130-131.

Sutor, D. J., and Wooley, S. E. (1974b). The organic matrix of gallstones. Gut, 15, 487-491.

Sutton, D. (1969). Textbook of Radiology, p. 720. Livingstone, Edinburgh. Williams and Wilkins, Baltimore.

Thistle, J. L., and Hofmann, A. F. (1973). Efficacy and specificity of chenodeoxycholic acid therapy for dissolving gallstones. New Engl. J. Med., 289, 655-659.

Thistle, J. L., and Schoenfield, L. J. (1971). Lithogenic bile among young Indian women: Lithogenic potential decreased with chenodeoxycholic acid. New Engl. J. Med. 284, 177-181.

Trotman, B. W., Petrella, E., Soloway, R. D., Sanchez, H., Morris, T., and Miller, W. T. (1974). Evaluation of radiographic lucency or opaqueness of gallstones as a means of identifying cholesterol or pigment stones: correlation of radio-opaqueness with mineral content. (Abstr.) Gastroenterology, 66, 791:

Wolpers, C. (1974). Morphologie der Gallensteine. Leber, Magen \& Darm., 4, 43-57. 